\title{
Reasons for the popularity of Tik Tok, the shortages, and the ways forward
}

\author{
Lizhao Dai ${ }^{1, a}$, Pei Chen²,b,*
}

${ }^{1}$ School of Journalism and communication in Jiangxi Normal University, Nanchang,Jiangxi,China

${ }^{2}$ School of Journalism and communication in Jiangxi Normal University, Nanchang,Jiangxi,China aemail: 1079702037@qq.com, bemail: 452376309@qq.com

${ }^{*}$ Corresponding author: Pei,Chen

Keywords: media social; media literacy; vulgar video; information mortuary;

\begin{abstract}
The popularity of Tik Tok has benefited from the overall development of the external short video industry, and it also relies on its own user positioning accuracy and functional perfection; but Tik Tok has obvious deficiencies in banning vulgar videos and avoiding user addiction, which requires not only Tik Tok platform improves the supervision system, and also requires users to continuously improve media literacy and cooperate with the regulatory authorities to work together to build a better approach.

\author{
抖音风靡的原因，不足及进路 \\ 戴利朝 $1, \mathrm{a}$,陈沛 $2, \mathrm{~b},{ }^{*}$ \\ 1江西师范大学新闻与传播学院, 南昌, 江西, 中国 \\ 2江西师范大学新闻与传播学院, 南昌, 江西, 中国 \\ aemail:1079702037@qq.com, bemail:452376309@qq.com \\ *通讯作者:陈沛
}

关键词：媒介社交；媒介素养；低俗视频；信息茧房；

摘要：抖音的风靡不仅得益于外在的短视频行业整体发展，还依赖于其自身用户定位精准及 功能完善; 但抖音在取缔低俗视频、避免用户沉迷等方面存在明显不足, 这不仅需要抖音平 台完善监管系统, 还需要用户不断提高媒介素养并配合监管部门, 共同努力建造一条更好的 进路。

\section{1.抖音风靡的原因}

\section{1 短视频行业崛起的带动}

2013 年，以秒拍、微视为代表的、立足于社交平台的短视频 APP 的出现，拉开了我国移 动短视频时代的帷幕。经过了几年的发展, 短视频 APP 拥有了庞大的用户群。2016 至 2017 年，短视频市场进入了所谓的爆发期，用户激增、市场快速扩大。2017 年，短视频市场规模 已经高达达 57.3 亿元，截止到 2018 年 2 月，短视频综合平台与短视频聚合平台活跃用户规 模分别达到 4.035 亿人与 1.655 亿人。在用户时间的占有上，短视频综合平台与短视频聚合平 
台的用户使用时长分别在 2 月份达到 51.628 亿小时、 16.167 亿小时的规模 ${ }^{1}$ 。, 抖音就是在 2016 年出现, 由此可见, 抖音是在短视频 APP 行业进入爆发期后开始登陆市场的, 这正说明抖音 的成功离不开短视频 APP 行业的崛起为其带来良好的外部环境。

\section{2 精准的用户定位}

抖音的设计团队在推出抖音之前，对短视频市场进行了非常深入的研究，他们认为当时 使用短视频 APP 的用户群体的组成很杂, 没有特征明显的使用群体。他们认为要想抖音在众 多短视频中异军突起, 需要有数量庞大且忠实的用户群作为支撑, 而广大年轻人作为互联网 中最活跃的群体，不仅数量众多而且对于传播能力最好易于扩大产品影响力。因此，抖音设 计团队将产品的用户定位于年轻群体。根据有关数据可以看到, 在抖音用户的年龄分布中, 24 岁及以下占比 27\%,25-30 岁占比 29.03\%,31-35 岁占比 $25.66 \%, 35-40$ 岁占比 $12.93 \%, 41$ 岁以 上只占 $5.37 \%$ 。证明 30 岁以下的年轻人占据了抖音用户数的绝大多数。由于年轻人喜欢音乐, 抖音非常有开创性的将年轻人与音乐结合在一起, 改变以往短视频多是用户原声的设计, 添 加音乐配乐功能, 这样他们就让抖音成为了一款属于年轻人的音乐短视频 APP。现如今, 打 开任一款音乐播放软件, 只要在搜索栏输入 “抖音” 都会出现一大串音乐作品, 而这些音乐作 品无一例外都在抖音有不少知名度, 许多音乐歌曲, 通过抖音这个传播媒介获取了一大批的 听众, 抖音为这些歌曲的宣传做出了巨大的贡献, 甚至可以说, 有不小部分的歌曲若不是因 为抖音, 就不会有如今的知名度。抖音团队充分挖掘抖音的市场潜质, 通过年轻群体进行传 播, 从而达到事半功倍的传播效果 ${ }^{2}$, 这充分证明了抖音的风靡离不开产品设计前期缜密的市 场调研和其正确的用户定位。

\section{3 完善的媒介社交系统}

截止 2018 年 12 月，我国网民规模达到 8.29 亿，互联网普及率达 59.6\%,手机网民规模达 到了 8.17 亿, 使用手机上网的比例提升至 $98.6 \%$ 。在互联网时代, 人们热衷于借助互联网获 取信息并与人分享和交流。媒介社交伴随着互联网的蓬勃发展已成为广大网民生活中不可或 缺的一部分。社交属性对于产品和使用者来说是非常重要的, 甚至可以说社交系统做的好不 好直接影响一款软件的受欢迎程度, 而抖音虽然是一款短视频传播软件, 但是它也设置了一 套非常完善的社交程序，让用户在观看和发布短视频之余也能与其他用户进行互动和交流， 满足用户的社交需求。4

打开抖音，在任何一条短视频作品界面的右边部分的界面排版从上到下依次是发布者的 头像、点赞图标、评论图标、分享转发图标; 其中，除了点赞之外的其他三项都具有很强的 互动社交属性，它们的作用如下：点击发布者的头像可以进入到发布者的个人主页当中，在 这里可以看到发布者的个人介绍、获赞总数、发布者关注的人、粉丝数、个人所有作品、所 有动态、发布者点过赞的所有作品, 可以在关注发布者后与其私信。这类似微博之类的个人 社交主页, 受众可以凭借这些数据轻易的分析出发布者的状态、个人喜好等等, 受众如对发 布者感兴趣，就可以与发布者进行私人联系。

点击评论图标可以看到所有对此作品进行过评论的用户的头像和他们的评论，并且还可 以这些评论进行点赞和评论, 这是个非常强大的互动网络, 评论者与发布者、评论者与评论 者之间进行互动, 这与微博的留言区的功能一样, 具有了极高的社交属性, 并且评论的内容 质量往往很高, 高赞评论往往自带笑点、自带剧本, 又能吸引其他人参与这条评论, 所以常 出现“我早晚要笑死在抖音的评论里”“笑死我对你有什么好处”之类的评论。

\footnotetext{
1易观. 2018 年中国短视频行业年度盘点分析 [EB/OL]. https://www. useit. com. cn/thread-18467-1-1.html, 2018.03.28 2李慧颖. 抖音 APP 的传播依赖研究 [J]. 中国报业, 2018(02) :45-46.

${ }^{3}$ CNNIC 发布第 43 次《中国互联网络发展状况统计报告》.

http://cnnic.cn/gywm/xwzx/rdxw/20172017_7056/201902/t20190228_70643.htm

4付浩. 基于社交网络结构的用户建模 [D]. 中国科学技术大学, 2016 .
} 
点击分享转发图标钮后，用户可以转发作品给好友、微信、朋友圈、QQ 空间等，这些 功能可以让用户向自己的“熟人圈”进行信息传播, 进行又一轮的社交互动, 继而带动更多人 喜爱抖音。抖音上这些功能的存在让用户在接收有趣的短视频信息的同时还可以满足他们的 社交需求，这便是抖音能如此受欢迎的一个重要原因。

\section{2.发展中出现的不足}

任何一款优秀的软件在发展过程中免不了出现一些缺点, 抖音也不例外, 笔者结合自身 的长期用户体验, 对五位爱使用抖音 APP 的同学、朋友分别进行了访谈, 在为了解抖音存在 哪些让用户觉得不满这个环节时，笔者给出了“缺乏创意的模仿内容太多”、“作品内容低俗 化”“歌曲配乐与视频内容不搭”、“缺少正能量的宣传”太太容易让人沉迷”这几个备选答案，可 以单选也可以多选并且允许访谈对象补充额外答案, 结果显示, 有一项五位访谈对象都选择 了，它是“容易让人沉迷”; 有四位访谈对象选择的是“作品内容低俗化”、有两位访谈对象选 择的是“缺乏创意的模仿内容太多”，其他两项“歌曲配乐与视频内容不搭”、“缺少正能量的宣 传”没有人选且也没有人给出其他答案。这个调查结果也与笔者的切身经验基本吻合, 能推断 出抖音存在两个方面的不足，一是内容低俗化，二是防沉迷措施不完善。

\section{1 存在低俗视频}

一篇以湖南省 303 位大学生为问卷调查采访对象的研究抖音 APP 的文献中有研究到, 当 提问受访者觉得抖音 APP 需要在哪里进行改进时, “降低内容的恶俗性、提高视频内容监管” 这一选项被选择的次数最多, 占比达到 $66.1 \%$ 。5这可以说明, 对于低俗视频内容的不满已在 抖音主力用户圈中拥有了不小的共识。

所谓低俗视频作品，主要是指以恶搞、偷拍、扰乱他人等不符合社会公序良俗甚至违反 法律的视频内容。低俗视频不仅存在于抖音中，也存在于短视频 APP 中，但由于抖音的火爆 及用户基数大让其在抖音中很常见。这有两个方面的原因：一方面由于抖音的用户定位是年 轻群体, 年轻人胆子很大, 追求刺激, 敢于不走寻常路, 但往往缺乏必要的规范意识, 在追 求曝光的心理作用下拍出低俗视频，而由于受众也多少年轻人，容易点赞此类视频甚至二次 传播, 这又引起了其他想拍出能“爆火”的视频的人跟风拍摄, 另一方面原因是抖音平台对此 类内容的监管措施不够严厉。

\section{2 防沉迷措施不够完善}

抖音运用大数据算法，基于用户基本信息、社交关系和用户的点赞互动、@好友、视频 发布等一系列行为, 做出精确地用户画像, 从而推荐更符合该用户偏好的内容, 为每位用户 打造个性化的世界。这就像是一个抖音世界里的“信息茧房”，信息茧房的概念由哈佛大学教 授凯斯·桑斯坦在其著作《信息乌托邦一一众人如何生产知识》中提出, 桑斯坦指出, 在信息 传播中，因公众自身的信息需求并非全方位的，公众只注意自己选择的东西和使自己愉悦的 通讯领域，久而久之，会将自身桎梏于像蚕茧一般的“茧房”中 ${ }^{6}$ ，“信息茧房”效应，可能会走 向崇尚开放、互动、多元的互联网精神的反面—即信息封闭、信息偏见和算法专制。7

抖音用户身处这个茧房之中，受制于这种算法专制，沉迷于自己喜欢的视频类型，面对 源源不断的感兴趣的视频, 常常无法自拔, 时间也在此一点点流逝, 因此常有人调㑆自己或 他人: “抖音五分钟, 人间一小时”。

2018 年 7 月，抖音推出了旨在推进青少年健康成长的“向日葵计划”，设置“实名验证+人

\footnotetext{
5 王超群, 邱玥, 王世茜. 从大学生 “抖音” 使用体验看移动短视频行业的发展策略 [J]. 视听, 2018 (12) : 148-149.

6凯斯·桑斯坦.信息乌托邦[M].北京：法律出版社，2008： 15.

7范红霞,孙金波.大数据时代算法偏见与数字魔咒——兼谈“信息茧房”的破局[J].中国出版,2019(10):60-63.
} 
脸识别 $+人 工$ 审核”三道防火墙、禁止未成年人使用直播、充值、打赏、提现等功能，之后又 在 2019 年进行了升级, 在原有功能的基础上, 未成年用户在推荐首页只能汶览由抖音青少年 内容团队特别精选的短视频。并且青少年模式用户在每天 22 时至次日 6 时期间被禁止使用抖 音。从此措施中可以看到国家对青少年在互联网时代身心健康发展的重视，也看到了抖音响 应政策、承担主体责任的担当，但是以上措施还有明显不足之处，首先，青少年模式是在打 开抖音时弹出的一个选项, 如果用户不主动点击就不会进入到此模式中, 其次, 抖音的登录 方式颇多, 微信、QQ 号、微博、手机号等方式都能登录, 能找到足够多的方法正常使用抖 音。

如果说抖音对于未成年人防沉迷措施做的还不错，那么对于成年人防沉迷来说，抖音就 做的远远不够，只是在 2019 年加入 “时间限制”和“风险提示”两个功能。用户连续使用 90 分 钟后, 正在播放的短视频上会出现一行文字, 提醒用户注意休息; 用户可以主动开通时间锁 功能, 由用户设定密码开启后一旦单日使用时长累计达到预设时间, 系统将自动锁定, 用户 需要重新输入密码才能继续使用。对于用户来说, 这个防沉迷系统约束能力太弱。提醒的内 容先用户完全可以当做没看见轻松刷过去, 而时间锁需要用户主动设置, 这对于抖音成瘾的 用户来说, 他会去设置的几率很小, 而对于其他用户来说, 就算设置了, 到提醒时间了, 要 还想刷也只用输入一次密码即可。

\section{3.抖音持续发展的进路}

要想改进这些不足, 让抖音始终能为用户带来良好的客户体验, 走出一条持续发展的进 路，离不开用户和平台以及监管部门的共同努力。

\section{1 平台完善监管系统}

首先, 抖音对这些不足要高度重视, 立足长远, 不可为了一时的流量而放任低俗类视频 的传播; 专门对低俗视频设立完善的监管机制, 对于用户所上传的视频要进行高质量的检测, 发现有低俗视频, 后台系统能及时辨别, 并且要加大人工复审的力度, 对不能分辨清楚的及 时进行人工复审。当确认是低俗视频时必须绝不姑息，及时对发布者进行警告、封号等处理;

同时, 要坚持不断的探索更加合理、可操作性更强的防沉迷系统，并且转变观念，重视 对于成年人用户的防沉迷保护。在流量经济的时代, 用户数量、在线时长、点击量就意味着 经济效益, 但是作为流量平台, 当遇到经济效益与用户身心健康需要二选一时, 应当毫不犹 豫、不遗余力的选择后者、支持后者。

\section{2 提高用户媒介素养}

美国媒介素养研究中心将媒介素养定义为“人们面对媒体各种信息时的选择能力、理解能 力、质疑能力、评估能力、创作和生产能力以及思辨的反映能力。”8对于抖音用户来说, 他 们在进行内容生产时坚持正确的价值观、不为了点赞而拍出低俗视频, 在观看视频, 一定要 有对视频内容最基本的鉴赏能力, 知道什么是低俗视频, 并且不要为了追求刺激、好玩而点 赞、留言互动或者进行二次传播。

抖音日常宣传工作要做到位, 让用户明确知道哪些视频是不能上传的; 鼓励用户举报此 类视频，号召用户积极参与到日常视频的监管中来，帮助用户提高媒介素养。

用户也应该自觉提高自己的媒介素养, 并要注意保护自己身心健康, 抖音虽好, 但毕竟 是电子产品, 一旦长时间使用会影响身心健康, 也会严重影响日常工作。并且根据笔者体验, 许多用户都有凌晨使用抖音而失眠的情况, 长此以往, 很容易造成健康问题。因此, 广大用 户一定要提高媒介素养、培养正确的使用习惯。毕竟防沉迷不能完全指望平台给力, 自身控 制也同样重要。

8 张开.媒体素养教育在信息时代[J].现代传播, 2003 (01): 116-118. 


\section{3 监管部门做好“把关人”}

相关监管部门要增强总体内容把关能力，强化信息获取、筛选和分析能力，及时更新有 利于抖音等互联网信息产品持续发展的政策和法规, 进一步探讨制定旨在保护互联网用户身 心健康的可行性方案，加强对抖音的监督，督促防沉迷措施的完善。做好在互联网中维护法 律法规、维护社会公序良俗、保护互联网用户身心安全的不大显眼但却最重要的“把关人”。

\section{4.结语}

抖音的出现与发展，壮大了短视频行业的市场规模，创新了短视频行业的发展模式，引 领了短视频行业的发展趋势, 也为我们广大用户提供了更优质的媒介体验服务, 这是非常值 得称道的。因此, 抖音一定要珍惜成果、保持成绩, 重点关注并努力尝试解决现存的各种不 足, 并要依靠用户、主动配合监管部门, 三方一道努力, 重视维护健康的互联网环境、重视 保护用户的身心体验，让抖音走在一条可持续发展的良好道路上。

\section{References}

[1] Yi Guan. 2018 China Short Video Industry Annual Inventory Analysis : https://www.useit.com.cn/thread-18467-1-1.html,2018.03.28

[2] Li Huiyin. Research on Propagation Dependence of Tik Tok APP[J]. China Newspaper Industry,2018(02)

[3] The 43rd Statistical Report on the Development of China's Internet Network: Http://cnnic.cn/gywm/xwzx/rdxw/20172017_7056/201902/t20190228_70643.htm

[4] Fu Hao. User modeling based on social network structure [D]. University of Science and Technology of China, 2016.

[5] Wang Chaoqun, Qiu Yi, Wang Shizhen. The development strategy of mobile short video industry from the experience of college students [J]. RADIO\&TV JOURNAL,2018(12):148-149.

[6] Keith Sunstein. Information Utopia [M]. Beijing: Law Press, 2008: 15.

[7] Fan Hongxia,Sun Jinbo.Algorithm Prejudice and Digital Magic in the Age of Big Data_Alsoon the Breaking of "Information Marriage"[J].China Publishing Journal,2019(10):60-63.

[8] Zhang Kai. Media Literacy Education in the Information Age [J]. Modern Communication, 2003 (01): 116-118.

[9] Luo Wei. The Status Quo and Trend of Short Video Development in China[J]. Electronic Technology and Software Engineering, 2018(22): 4.

[10] Deng Chuchu. From the short video of vibrato to see the social communication of video entertainment [J]. New Media Research, 2018, 4 (22): 120-121. 